\title{
EFFECT OF CHEMICAL TREATMENTS ON THE
}

\section{CHARACTERISTICS OF REGULAR AND COMPACT COTTON SPUN} YARNS

\author{
Afrose Fathima Farid ${ }^{1}$, Roshanara ${ }^{2}$, V. Subramaniam ${ }^{3}$ \\ ${ }^{1}$ Department of P.G. Studies \& Research in Home Science, Textile Science \& Fashion Designing, Justice Basheer \\ Ahmed Sayeed College for Women, Teynampet, Chennai 600018 \\ ${ }^{2}$ Department of P.G. Studies \& Research in Home Science, Justice Basheer Ahmed Sayeed College for Women, \\ Teynampet, Chennai 600018 \\ ${ }^{3}$ Department of Textile and Technology, Jaya Engineering College, Chennai 602024
}

\begin{abstract}
An investigation on the physical properties of compact and conventional spun yarns following chemical treatments is reported. In this research, the effect of chemical finishing treatments namely, scouring, hypochlorite bleaching, peroxide bleaching, mercerising and dyeing on the yarn characteristics of $30 \mathrm{Ne}$ regular and compact ring spun yarns has been studied. The results showed that whilst tenacity of treated yarns rose, elongation fell with the exception of slack mercerising treatment. Work of rupture was significantly higher than that of control for slack mercerised samples. With the exception of slack mercerised yarns there was no measurable difference in initial modulus of yarns. While bleached yarns samples showed somewhat poor abrasion resistance, the other treated yarns did not show any change in abrasion resistance. All the treatments had led to an increase in yarn friction. Slack mercerisation treatment had led to a significant increase in shrinkage and the shrinkage values were identical in both conventional and compact yarns. The other treatments had resulted in lower shrinkage values. Compression was found to be greater for compact yarns in untreated state and all the treatments had lowered the compression. Compressional energy (WC) was found to be the highest for compact mercerised yarn. Scanning electron micrographs showed clearly the improved hairiness and bulk in compact yarns.
\end{abstract}

Keywords: Pneumatic Compact Spinning, Conventional Ring Spinning, Yarn Physical Properties, Kawabata Analysis, Scouring, Hypochlorite Bleach, Hydrogen Peroxide Bleach, Mercerization, Reactive Dyes.

\section{INTRODUCTION}

Spinning is the process of creating yarn from various raw fibre materials. Separate fibres are twisted together to bind them into a stronger long yarn. Characteristics of the yarn vary based on the material used, fibre length, alignment, quantity of fibre used and degree of twist ${ }^{[1]}$

In conventional ring spinning, the zone between the nip line of the delivery rollers and the twisted end of the yarn is called the "spinning triangle". This is the most critical part of the ring spinning system. In this zone, the fiber assembly doesn't have any twist. The edge fibers ply out from this zone, and make little or no contribution to the yarn tenacity. Furthermore, they lead to yarn hairiness. ${ }^{[2]}$ In compact spinning, the "spinning triangle" is eliminated and almost all fibers are incorporated into the yarn structure under the same tension. This leads to significant advantages such as increasing yarn tenacity, yarn abrasion resistance and reducing yarn hairiness. ${ }^{[3,4]}$

Yarn manufactured by means of the compact spinning system compared with classical yarn is characterised by better smoothness, higher luster, better abrasion fastness, lower hairiness higher tenacity and elongation at break and smaller mass irregularity Compact yarns are claimed to be stronger and less hairy due to the improved fibre binding and have better yarn elongation, work capacity and less yarn irregularity compared with conventional ring yarns. ${ }^{[5]}$ As a result, these yarns have tremendous potential to offer several advantages both in spinning and in all subsequent processing stages compared to conventional ring yarns. The better hairiness, tenacity and elongation properties of compact spun yarn provide opportunities to work with lower twist coefficient, resulting in an increase in production rate and better properties in the end product ${ }^{[6]}$

Because of the improved yarn structure by compact spinning, there are a number of designs in the market. The most popular models are Riter (Comfor Spin), Zinser (AirComTex 700) and the Süessen (EliTe Spinning) systems. These systems are somewhat different in each case, but all of them are based on the same principle of the "elimination" of the spinning triangle by pushing the staple fibres together, or condensing them to attain a much smaller spinning triangle than with conventional ring frames ${ }^{[7]}$ For instance, while in Air-Com-Tex 700, a perforated apron is added to the classical drafting system of the ring machine, whereas Comfor-Spin uses a perforated drum to condense 
fibres in the drafting region. On the other hand, the EliTe system from Süessen differs significantly from both systems. $^{[8]}$

The paper by Atlas et. al. ${ }^{[2]}$ dealt with the comparison of conventional ring mechanical compact and pneumatic compact yarn spinning systems. Many of the compact spinning systems use perforated drums or lattice aprons over the openings of the suction slots. With the air flow, the fibers move sideways and they are consequently condensed. Mechanical Compacting systems also used and has some attractiveness in that the energy consumption is less.

Compact yarns have tremendous potential to offer several advantages both in spinning and in all subsequent processing stages compared to conventional ring yarns. The characteristic properties of the spun yarn have significant influence on the performance of subsequent fabric manufacturing operations as well as the end-product quality ${ }^{[9]}$ Whenever a new spinning technology is launched in the textile market, examining and defining the new yarn performance become obligatory.

A survey of literature on compact yarns showed the absence of published work on the effects of chemical treatments on the yarn characteristics. Hence this research is concerned with the comparison of physical and mechanical properties of chemically treated and untreated conventional and compact ring spun yarns of $30 \mathrm{Ne}$ counts.

Subjecting the yarns to these preparatory processes and studying their properties subsequently would provide a better insight into the behaviour of fabrics at the processing stages. This will also serve to foresee the performance of fabrics made from these yarns in wet processing and to take such actions as are warranted.

\section{MATERIALS AND METHODS}

In the first part of the study a mixing of Shankar- 6 cotton fibres with an average length of $30.3 \mathrm{~mm}$ and 3.8 micronaire were spun into compact yarn and conventional yarns of 30 Ne using the Süessen EliTe compact spinning machine and Lakshmi Rieter G 5/1 ring spinning machine. The measured twist per inch for the conventional spun yarns was 20 and the compact yarn was 18.2.

In the second part the conventional and compact ring spun yarns of $30 \mathrm{Ne}$ count were subjected to a series of finishing treatments - scoured, mercerised, bleached and dyed following the industrial practices. The finishing treatments given are graphically represented in the form of a flow chart.

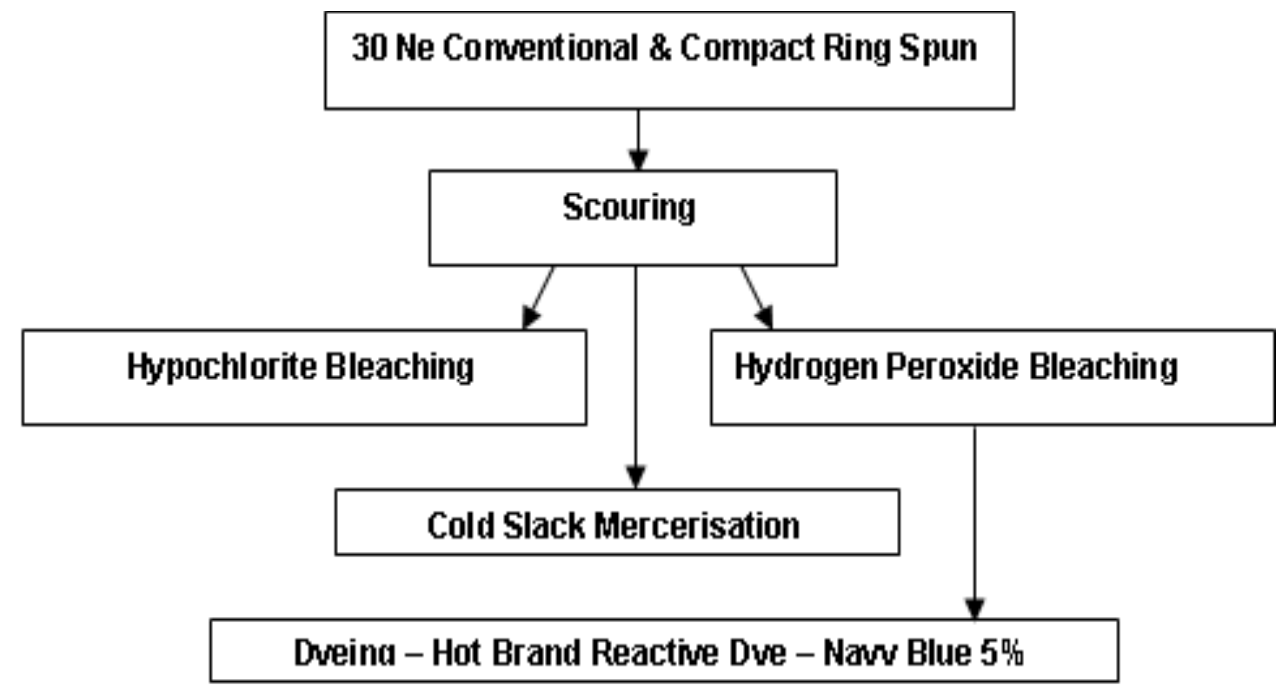

Fig 1 Process Flow Diagram of the Chemical Finishing Treatments

In the third part of the study the yarn samples were tested using various instruments.

\subsection{Single Yarn Strength}

The Uster Tensorapid 3 and Textechno Statimat M Tensile Tester were used for yarn testing. Characters such as the breaking strength, breaking elongation, breaking tenacity, work of rupture, breaking force and the work done to break the yarn were recorded. The mean of 100 readings was taken. The ASTM Standard D2256 - 97, standard test method for tensile properties of yarns by the single-strand method was followed.

\subsection{Initial Modulus}

The initial modulus value were obtained by taking the slope of the stress strain curve from the Instron Tensile Tester values and expressed as $\mathrm{g} / \mathrm{tex}$.

\subsection{Yarn Appearance}

The irregularity values such as percentage mean deviation, Uniformity\% (U\%), coefficient of variation commonly known as $\mathrm{CV} \%$ and the yarn hairiness index $\mathrm{H}$ were obtained using the Uster Evenness Tester $4 \mathrm{SX}$ at $400 \mathrm{~m} / \mathrm{min}$. The ASTM Standard D 1425 - 96, standard test method for unevenness of textile strands using capacitance testing 
equipment was followed. The sensors note the number of imperfections exceeding predetermined levels of average yarn diameters and ten tests per sample were evaluated.

\subsection{Yarn Quality Index}

The Yarn Quality Index (YQI) was calculated using Barella's ${ }^{[9]}$ formula as shown below:

\section{YQI $=\underline{\text { Yarn Tenacity } x \text { Elongation }}$

$$
\mathrm{U} \%
$$

\subsection{Yarn Diameter}

The yarn diameter was measured optically. The yarn was passed between an optical sensing head and a light source. Light was projected on one side of the yarn and is either blocked by the yarn or received by the pixel in the camara (CCD array). The pixels that received light are considered light pixels while the ones that do not receive light are considered dark pixels. The yarn diameter is calculated as the distance between the first dark pixel ' $\mathrm{D}$ '.

\subsection{Yarn Hairiness}

The Uster Tester $4 \mathrm{SX}$ was used to determine the yarn hairiness $(\mathrm{H})$ optically in terms of different lengths of protruding fibres on the yarns. The number of protruding fibres in the Ne 30 compact and conventional yarns were counted and expressed as mean hairiness value.

\subsection{Scanning Electronic Microscopy (SEM)}

The scanning electron microscope (SEM) is a type of electron microscope capable of producing high-resolution images of a sample surface. The yarn structure and surface of the compact and conventional ring spun yarns were analysed microscopically at various magnifications such as $100 \mu$ and $150 \mu$.

\subsection{Yarn to Metal Friction}

In a yarn, it is preferable to have a good inter-fibre friction so that the tensile and other yarn properties improve. The ASTM D3412-95, standard test method for coefficient of yarn friction was followed and the Lawson Hemphill Constant Tension Transport (CTT) was used to measure the yarn to metal friction. The coefficient of friction was measured. Ten tests were carried out for assessing the friction coefficient of yarns.

\subsection{Abrasion Resistance of Yarn}

The ASTM D1379-64 standard method for testing abrasion resistance of single yarn was followed. The MAG SITRA computerised yarn abrasion resistance tester was used. The number of strokes required to break for each yarn was recorded. Ten readings per yarn abraded against emery 600 at a pretension of $20 \mathrm{~g}$ were recorded.

\subsection{Yarn Compression}

Yarn compression tests were conducted on the Kawabata evaluation system. Here, a standard area of the yarn was subjected to a known compressive load and then the load was gradually relieved. The following mechanical parameters characterize the compression and recovery behaviour of the yarn:

$\mathbf{T}_{\mathbf{0}^{-}} \quad$ Yarn thickness $(\mathrm{mm})$ at a very low compressive stress of $0.5 \mathrm{gf} / \mathrm{cm}^{2}$

$\mathbf{T}_{\mathbf{m}^{-}} \quad$ Yarn thickness at a maximum compressive load of $50 \mathrm{gf} / \mathrm{cm}^{2}$

WC- Work done in compression represented by the area under the compressive curve.

RC- Compressive resilience is the work recovered to the work done, expressed as a percentage.

LC- Compression linearity is a measure of the deviation of the deformation curve from a straight line. Higher value of LC implies a higher initial resistance to compression.

\section{RESULT AND DISCUSSION}

\subsection{Yarn Tenacity (Rkm)}

The tenacity values have been represented diagrammatically in Figure 2. It can be noticed that the compact yarns continue to maintain their trend in tenacity as compared to regular yarns with the exception of the hydrogen peroxide bleached and mercerised samples.

The tenacity of the scoured compact yarn was significantly higher than that of its counterpart regular yarn by $11.2 \%$. The removal of impurities and further alignment of fibres in the yarn structure due to the scouring wet treatment have resulted in the further increase in its tenacity in the case of compact yarn. According to Tripp et.al., ${ }^{[11]}$ The increase in the tenacity value of scoured samples is attributed to the removal of impurities in the yarn structure, which results in increase in yarn quality in terms of strength and elongation.

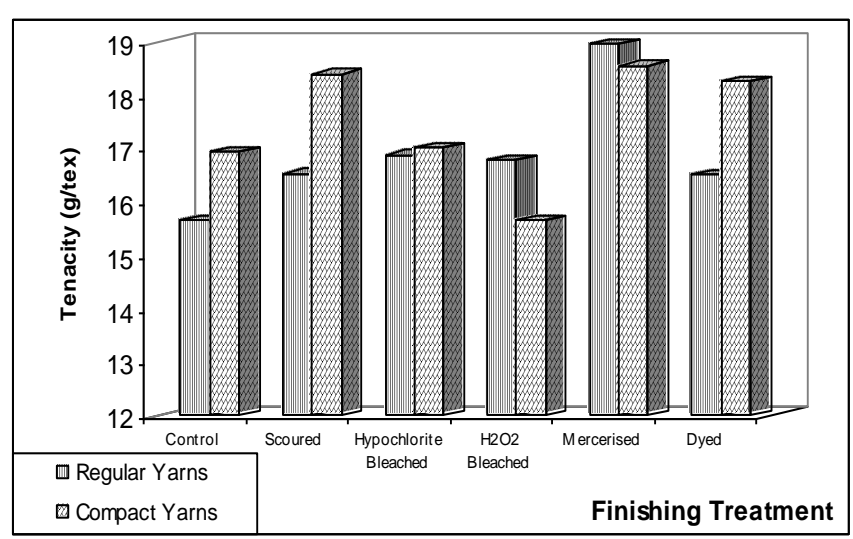

Fig 2 Effect of Chemical Treatments on Yarn Tenacity

The hypochlorite bleach treatment increased the strength of both the yarns. The peroxide treatment also reduced the tenacity of compact yarn. Hydrogen peroxide bleaching treatment being a degradative treatment has brought down the yarn quality by lowering the strength of compact yarn. 
The slack mercerisation treatment significantly increased the strength of both the compact and regular ring spun yarns. This is in agreement with the findings of Cheek and Roussel's ${ }^{[12]}$ study on the effect of mercerisation of flax and cotton yarns.

The regular yarn that has been mercerised has higher tenacity value than that of the compact yarn. This may be due to the fact that a regular yarn has a higher propensity to swell in view of its loose structure. In contrast, the compact yarn is difficult to swell by the alkali owing to its compact nature.

A very interesting observation noticed was the significant increase in the strength of the compact yarn following dyeing as compared to the control yarn. There was an increase of $10.6 \%$ in tenacity in respect of the dyed compact yarn when compared to its counterpart. This may be due to the less dye uptake in respect of compact yarn compared to the regular yarn.

\subsection{Yarn Breaking Elongation}

A comparative study between the two yarns (Figure - 3) shows that the elongation percentage was higher in some cases for regular yarn and so also for compact yarn in some cases. The scouring treatment reduced the extension percentage of both the yarns.

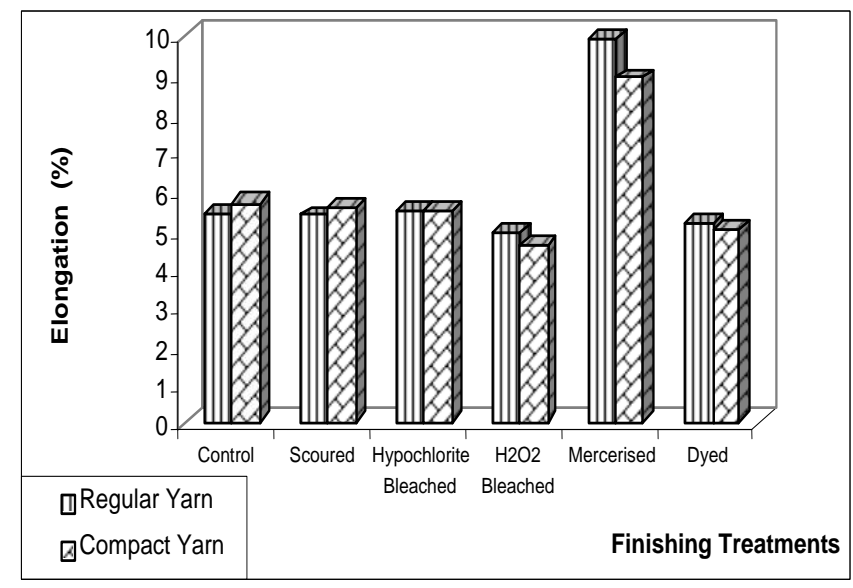

Fig 3 - Effect of Chemical Treatments on Yarn Elongation

Both the bleaching treatments due to the degradative action of bleaching agent lowered the elongation percentages of the compact yarn. The peroxide bleach lowered the elongation percentage of regular yarn.

The mercerisation treatment increased the elongation percentage by about 9.9 and 8.92 percent in the case of regular and compact yarns respectively. There are significant differences in the percentage values of the two yarns. This finding is in agreement with those of Rebenfeld ${ }^{[13]}$ who reported on the response of cotton fibres to chemical treatments. The increase in elongation percentage can be attributed to the shrinking and swelling of the fibres, which delay its breaking point.
Dyeing treatment reduced the elongation percentage of both the samples. This can be attributed to the covalent bond formation due to reactive dyes.

\subsection{Work of Rupture}

The breaking energy values of the untreated and the chemically treated conventional and compact ring spun yarns show that the scouring treatment increased the value of the compact yarn while the opposite effect was noticed in regular yarn. The increase in the breaking energy of the compact yarn can be attributed to the removal of impurities and further alignment of the compact yarn, thus increasing its strength. (Figure 4)

Both the bleaching treatments lowered the work of rupture values of both the yarns. Loss in work of rupture was noticed in the two yarns after hypochlorite treatment while that of hydrogen peroxide bleach treatment was found to be significant. The compact yarn had lower work of rupture value by $10.6 \%$ than its counterpart.

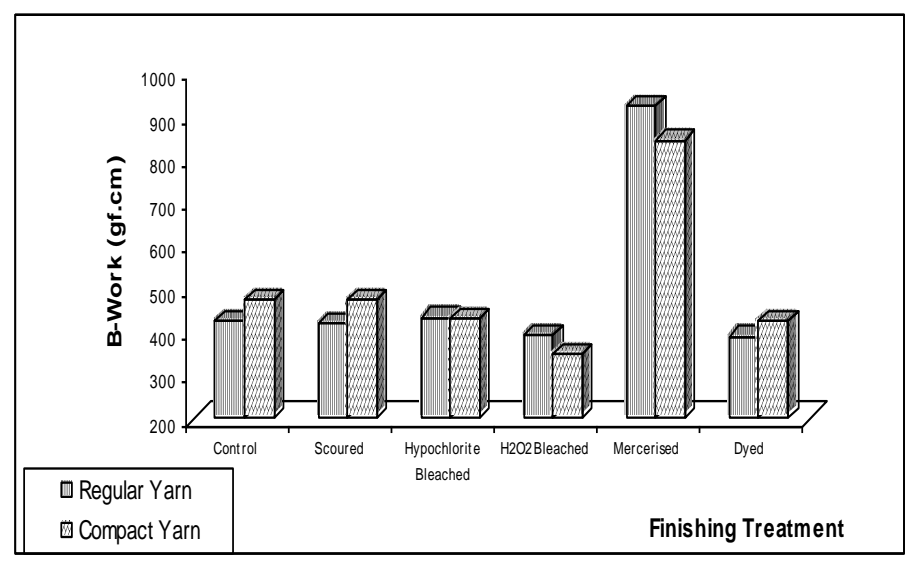

Fig 4 - Effect of Chemical Treatments on Yarn Work of Rupture

The effect of mercerisation treatment on the work of rupture, values of both the yarns were on positive side. The regular yarn had a better breaking energy value, which was higher than the compact yarn value by $8.3 \%$. The difference in the values of the two yarns following reactive dyeing was about $9.3 \%$. The compact yarn showed slightly better value than the regular yarn.

\subsection{Initial Modulus}

The initial modulus values calculated from the stress strain curve of the compact and regular yarn after chemical treatment are graphically represented in Figures 5. Examination of the initial modulus data of compact and regular yarns samples of treated regular and compact yarns shows some interesting results. Most of the samples namely the control, scoured, hypochlorite bleached, mercerised and dyed compact yarn samples show higher initial modulus when compared to their regular yarn counterparts. The percentage difference between the samples varied between 10 to $17 \%$. The peroxide bleached compact yarn sample 
showed a marginal difference of $3.6 \%$ compared with regular yarn. Initial modulus refers to stiffness of yarns.

Both the mercerised yarn samples exhibited a severe drop in their initial modulus when compared to their respective control samples. This finding is in agreement with that of Nayar ${ }^{[14]}$ who found that as the yarns become coarser, the initial modulus dropped. The reason for this drop in initial modulus of mercerised sample and the general higher initial modulus of most of the chemically treated compact yarn samples can be attributed to the fact that the drops as the yarn diameter becomes more.

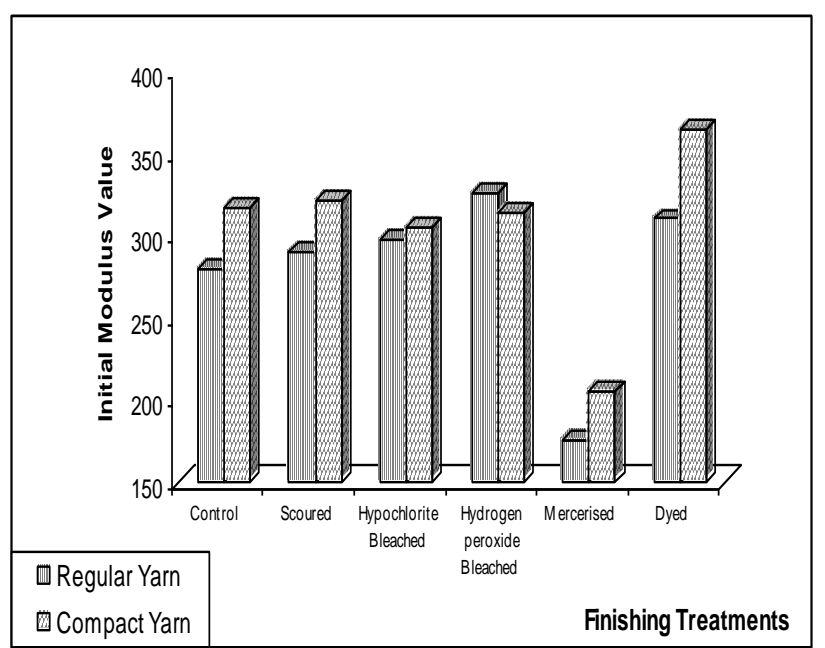

Fig 5 - Effect of Chemical Treatments on Initial Modulus

\subsection{Yarn Abrasion}

The abrasion values are plotted in Figure 6 from which, it can be inferred that the untreated regular yarn shows better abrasion resistance of abrasions cycles than the untreated compact yarn. Since the compact yarns surface is smoother, the yarn slips sooner and the cycle ends sooner. This finding is in agreement with that of Karthikeyan. ${ }^{[15]}$ It is interesting to note that the abrasion value of conventional yarn decreased following scouring, while opposite was the trend in the compact yarn for the same treatment. The scoured regular yarn had the value 53.33 cycles, while the compact yarn counterpart had the value of 50.4 cycles.

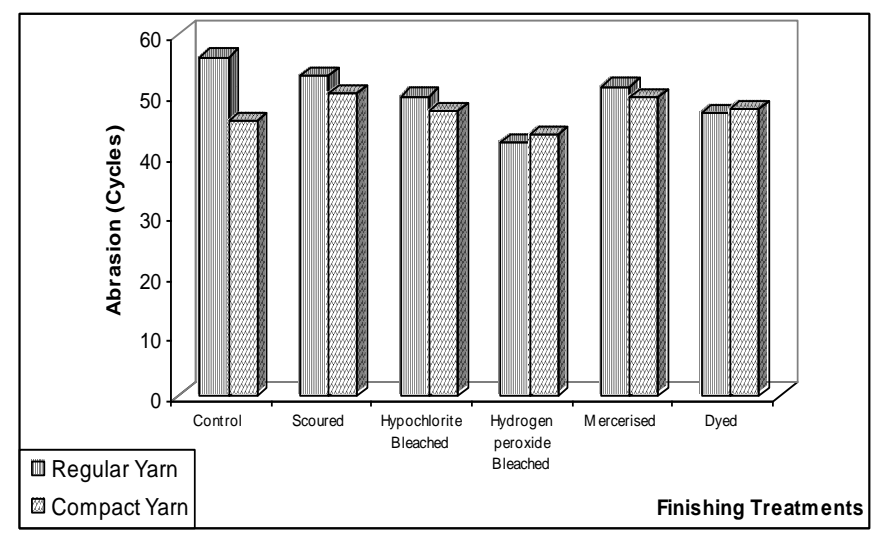

Fig 6 Effect of Chemical Treatments on Yarn Abrasion
The chlorite bleach, reduced the number of abrasion cycles in the regular yarn. The trend was opposite in the case of the compact yarn. Lower abrasion resistance was noticed in the case of both the yarns when treated with peroxide bleach.

The mercerisation treatment resulted in increase in the abrasion value of the compact yarn while a reduction was noticed in regular yarn. According to Imayathanizhan and Gajendra ${ }^{[16]}$ the reason for the increase in the abrasion value after $\mathrm{NaOH}$ treatment can be attributed to the degree of substitution of O-Na groups in the cotton fibre.

The dyeing resulted in decrease in abrasion value of both the yarns. The reason for the increase in abrasion resistance can be attributed to the extent of fibre swelling which occurs during the chemical processing. Because of the increased diameter, the yarn slippage time increases.

\subsection{Yarn Friction}

From Figure 7 it can be clearly noticed that all the treated and untreated compact yarn samples had lower friction values when compared to their respective regular yarn counterparts. The friction between yarn to metal should be as low as possible, for lower the yarn to metal friction, lower will be the yarn breakage rate in next processing and higher will be the product quality. ${ }^{[17]}$

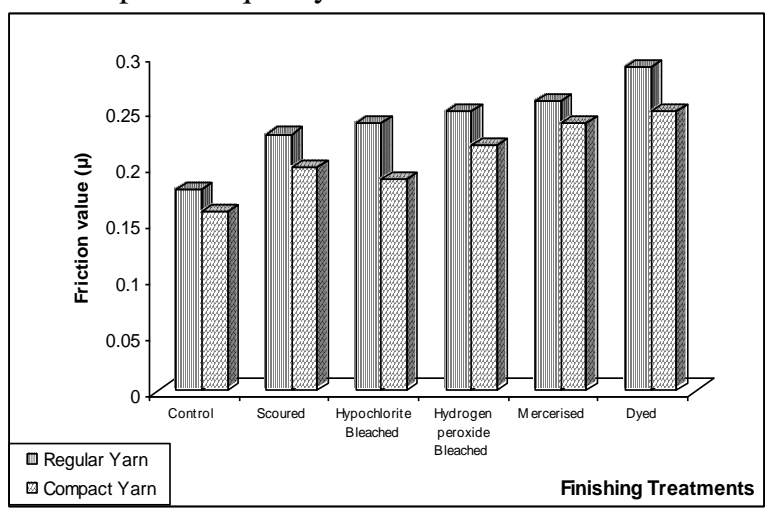

Fig 7 - Effect of Chemical Treatments on Yarn Friction

The untreated samples had the values 0.18 and 0.16 with regard to the regular and compact yarns respectively. The scoured regular and compact yarns had the values 0.23 and 0.2 . The hypochlorite regular and compact samples had the values 0.24 and 0.19 . The peroxide samples had the values 0.25 and 0.22 . The mercerised regular and compact yarns had the values 0.26 and 0.24 respectively. The dyed samples had the values 0.29 and 0.25 with regard to regular and compact yarns. The least value was noticed in untreated samples while the maximum was seen in dyed samples. These findings were in agreement with those of Ragunathan. ${ }^{[18]}$ This is due to the lower area of contact of the compact yarns.

An inverse relationship was noticed between the friction and abrasion values. The increase in friction values directly caused a decrease in abrasion values. The reason for the higher frictional value of the regular yarn than that of the compact yarn can be attributed to an increase in area of contact following chemical treatments. 


\subsection{Yarn Shrinkage}

Yarn shrinkage after different chemical treatments is shown in Figure 8. Scouring resulted in shrinkage of both the yarns. Both the yarn shrunk to the same length. The bleaching and dyeing treatments led to higher shrinkage for both the yarns. The shrinkage percentage of the regular yarn was 2.4 while that of the compact yarn was 1.49 percent for both the bleaching and dyeing treatments. The regular yarn exhibited more shrinkage after these treatments than the compact yarn counterparts.

The slack mercerised samples showed an exceptionally high shrinkage of 21.64 and 21.42 percent with regard to regular and compact yarns when compared with their respective control samples. The difference was found to be significant.

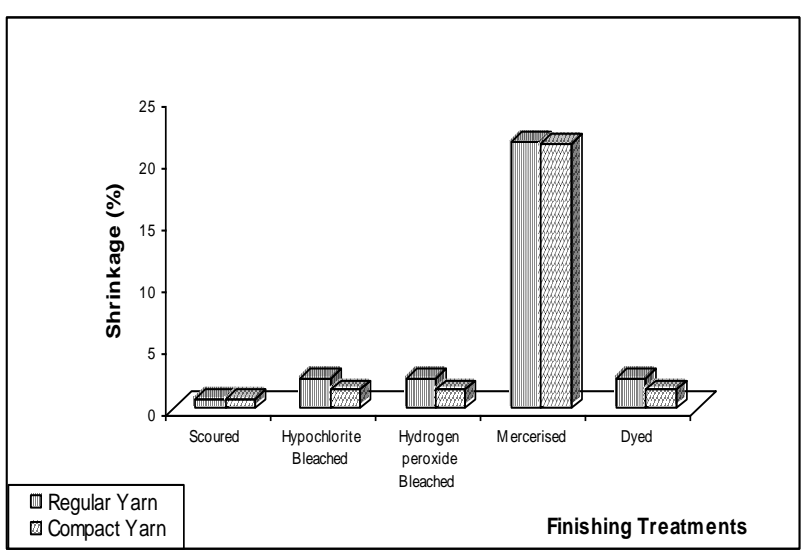

Fig 8 - Comparison of Yarn Shrinkage Percentage between Chemically Finished Regular and Compact Yarns

\subsection{Yarn Compression}

Table 1 and Figures 9 -14 give the compression properties of regular and compact yarns subjected to various chemical treatments.

Table 1 Compressional Values of Untreated and Chemically Treated Yarn

\begin{tabular}{|l|l|l|l|l|l|l|}
\hline Yarn Type & $\mathbf{L C}$ & $\begin{array}{l}\text { WC } \\
\text { units }\end{array}$ & $\mathbf{R C}$ \% & $\mathbf{T}_{\mathbf{o}} \mathbf{m m}$ & $\begin{array}{l}\mathbf{T}_{\mathrm{m}} \\
\mathbf{m m}\end{array}$ & $\mathbf{\%} \mathbf{C}$ \\
\hline Regular Control S & 0.444 & 0.132 & 36.36 & 0.239 & 0.12 & 49.79 \\
\hline Compact Control & 0.283 & 0.135 & 35.56 & 0.303 & 0.112 & 63.036 \\
\hline Regular Scoured & 0.456 & 0.139 & 38.13 & 0.249 & 0.127 & 48.995 \\
\hline Compact Scoured & 0.54 & 0.135 & 47.41 & 0.229 & 0.129 & 43.668 \\
\hline Regular Hypo Chlorite Bleached & 0.358 & 0.112 & 39.93 & 0.242 & 0.117 & 51.652 \\
\hline Compact Hypo Chlorite Bleached & 0.452 & 0.148 & 39.19 & 0.256 & 0.125 & 51.171 \\
\hline Regular Hydrogen peroxide Bleached & 0.395 & 0.147 & 34.01 & 0.276 & 0.127 & 53.985 \\
\hline Compact Hydrogen peroxide Bleached & 0.437 & 0.117 & 25.64 & 0.229 & 0.122 & 46.724 \\
\hline Regular Mercerised & 0.522 & 0.132 & 30.85 & 0.186 & 0.084 & 54.8 \\
\hline Compact Mercerised & 0.427 & 0.188 & 38.83 & 0.31 & 0.134 & 56.774 \\
\hline Regular Reactive Dyed & 0.466 & 0.142 & 39.44 & 0.271 & 0.149 & 45.018 \\
\hline Compact Reactive Dyed & 0.822 & 0.143 & 53.85 & 0.229 & 0.137 & 40.174 \\
\hline
\end{tabular}

In addition to the parameters provided by Kawabata compression tester, the parameter percent compression has been calculated using the following formula:

$$
\text { Percent compression } \quad=\quad \underline{T}_{0}-\frac{T_{m}}{T_{0}}
$$

Where, $\mathrm{T}_{\mathrm{o}}$ is the thickness of the yarn, measured at 0.5 $\mathrm{gf} / \mathrm{cm}^{2}$ and $\mathrm{T}_{\mathrm{m}}$ the thickness at $50 \mathrm{gf} / \mathrm{cm}^{2}$. It is apparent that considerable changes have occurred in the compressional properties of regular and compact cotton spun yarns. The compact control yarn was characterized by higher compression (63.1\%) vis-a-vis regular ring spun yarn $(49.8 \%)$.

All the treatments applied to the compact yarns have led to a drop in compression percentage. WC, which is the compression energy, shows a significant increase in slack mercerised compact yarns $(0.188$ as against 0.135 for control). Compressional resistance is the least for hydrogen peroxide bleached yarn. Slack mercerisation of compact yarns has led to an increase in percent compression.

The regular spun cotton control yarn shows a compression of $49.79 \%$ and bleaching by both hypochlorite and hydrogen peroxide treatments and slack mercerisation has led to a significant increase in its value. The percent compression has been found to be a useful measure of compression for fabrics and in the case of yarns also it is found to be a useful parameter to have an understanding of compression. WC values are different but there seems to be no trend in them.

It may be argued that $\mathrm{T}_{\mathrm{o}}$ values of regular and compact yarns show contradictory results in that the value in respect of 
compact yarn is higher than that of the control. It may be noted that $T_{0}$ values are the thickness of the yarn after compressing them under $0.5 \mathrm{gf} / \mathrm{cm}^{2}$ pressure. The real thickness of the yarns is the thickness at zero pressure, which is difficult to measure in the tester. Thus, it is noticed that the compact yarn is most sensitive to the treatments as reflected by the compression percentage. Compression of the yarn following scouring is due to the removal of the impurities. A decrease in the $\mathrm{T}_{\mathrm{o}}$ after scouring is a confirmation that the yarns have become more compact.

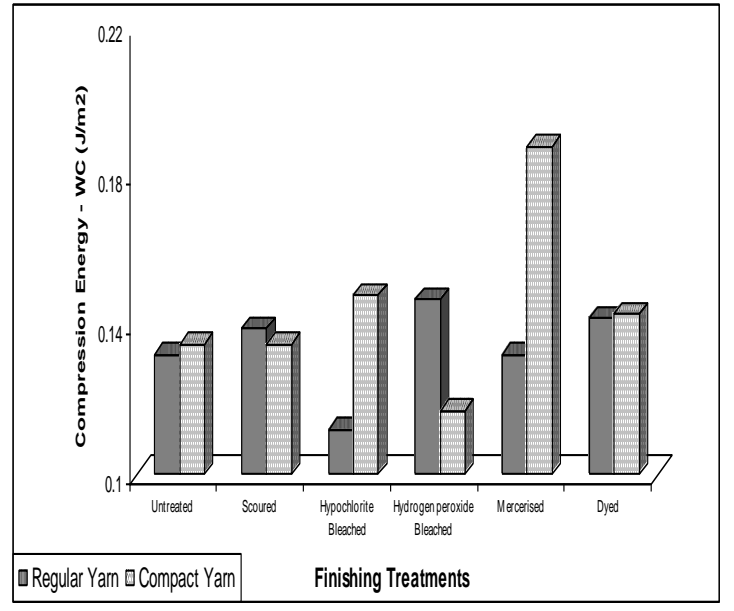

Fig 9 - Effect of Chemical Treatments on Yarn Linearity of Compression

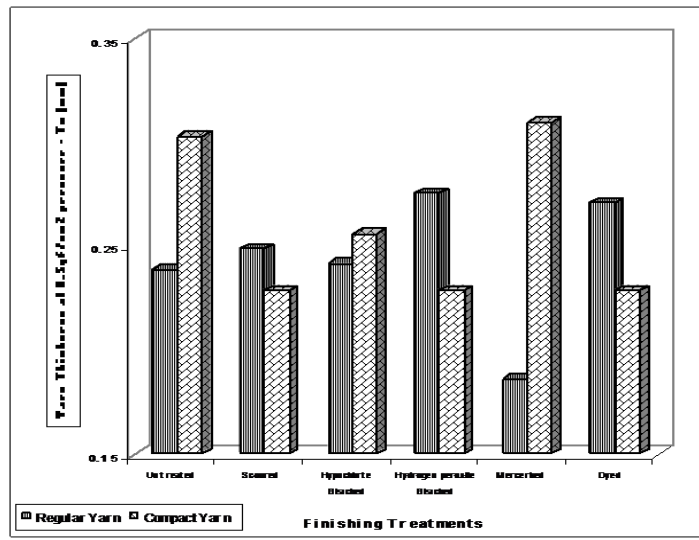

Fig 11 - Effect of Chemical Treatments on Yarn Compressional Resilience

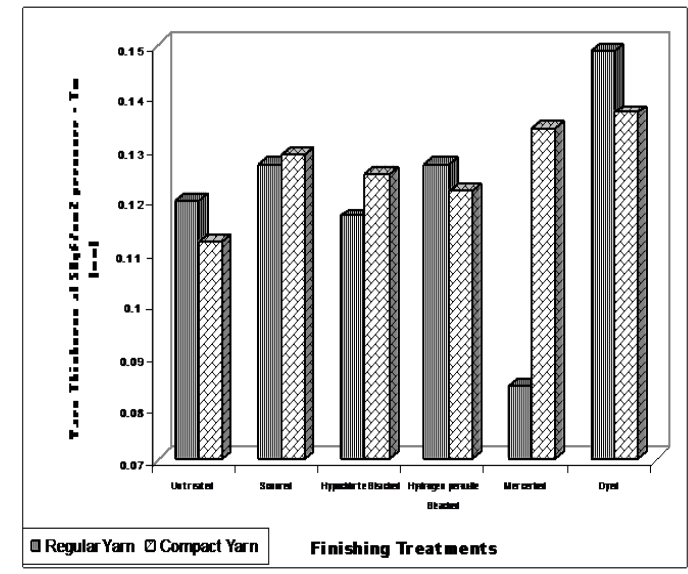

Fig 13 - Effect of Chemical Treatments on Yarn Thickness (Tm)

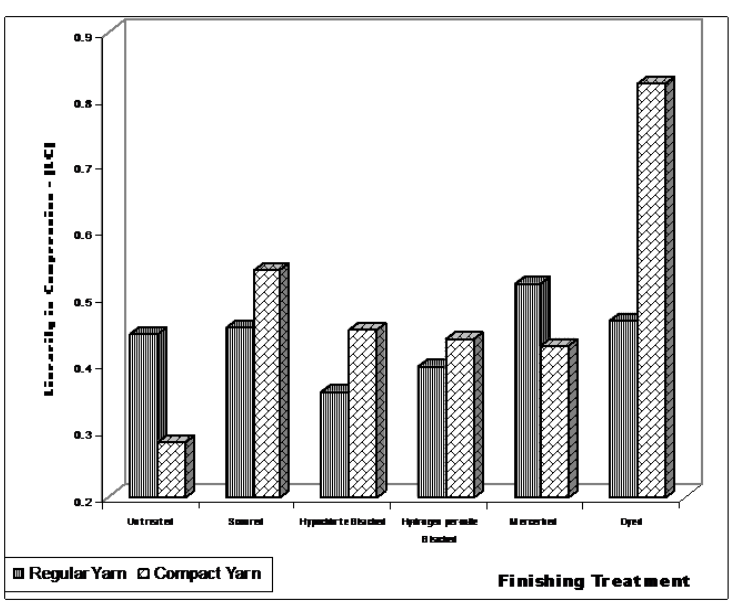

Fig 10 - Effect of Chemical Treatments on Yarn Compressional Energy

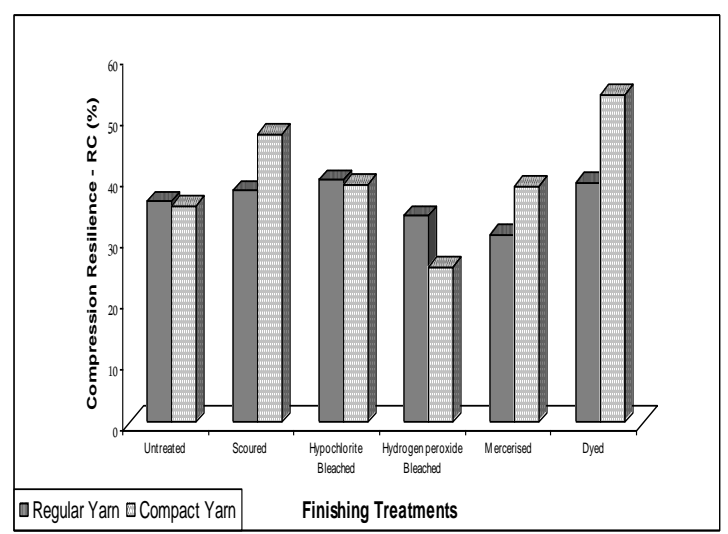

Fig 12 - Effect of Chemical Treatments on Yarn Thickness (To)

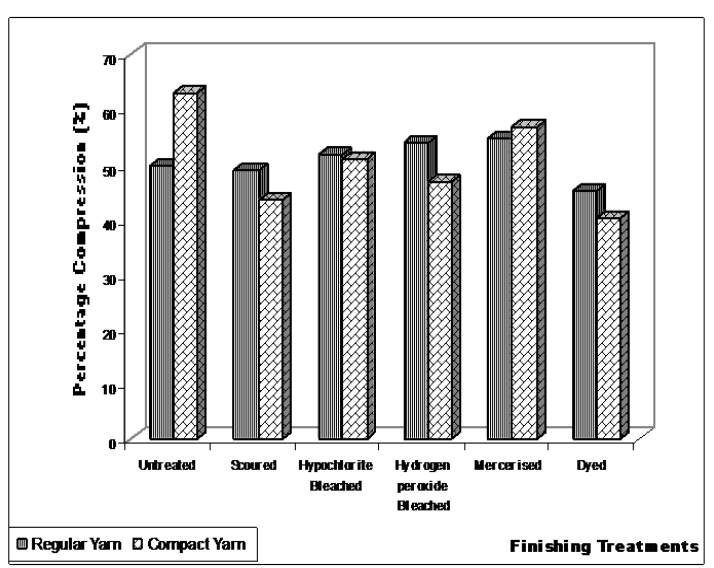

Fig 14 - Effect of Chemical Treatments on Yarn Compression Percentage 
It is the major axis, which is compressed during compression of yarns. Since WC is influenced by yarn thickness, ratio of $\mathrm{WC} / \mathrm{T}_{\mathrm{o}}$ which is normalized has been taken to represent compression. Table 2 shows the correlation matrix for compressional properties. LC is strongly correlated with $\mathrm{WC} / \mathrm{T}_{\mathrm{o}}$. $\mathrm{LC}$ is also well correlated with $\mathrm{C} \%$ negatively.

Table 2 - Correlation between the Compression Properties

\begin{tabular}{|l|l|l|l|l|l|l|l|}
\hline & $\mathbf{L}$ & $\mathbf{W}$ & $\mathbf{R}$ & $\mathbf{T}_{\mathbf{o}}$ & $\mathbf{T}_{\mathbf{m}}$ & $\mathbf{C \%}$ & $\begin{array}{l}\mathbf{W C} \\
\mathbf{T}_{\text {o }}\end{array}$ \\
\hline $\mathbf{L C}$ & 1 & $\begin{array}{l}.0 \\
\mathbf{C}\end{array}$ & .4 & -.500 & .229 & $\mathbf{- . 7 4 7}$ & $\mathbf{. 6 2 9}$ \\
\hline $\mathbf{W C}$ & & 1 & .2 & $\mathbf{. 5 9 3}$ & .372 & .222 & .380 \\
\hline $\mathbf{R C}$ & & & 1 & .304 & .747 & -.480 & -.216 \\
\hline $\mathbf{T}_{\mathbf{0}}$ & & & & 1 & .483 & .499 & -.501 \\
\hline $\mathbf{T}_{\mathbf{m}}$ & & & & & 1 & -.512 & -.263 \\
\hline $\mathbf{C \%}$ & & & & & & 1 & -.219 \\
\hline $\mathbf{W C} / \mathbf{T}$ & & & & & & & 1 \\
\hline
\end{tabular}

\subsection{Scanning Electron Microscope}
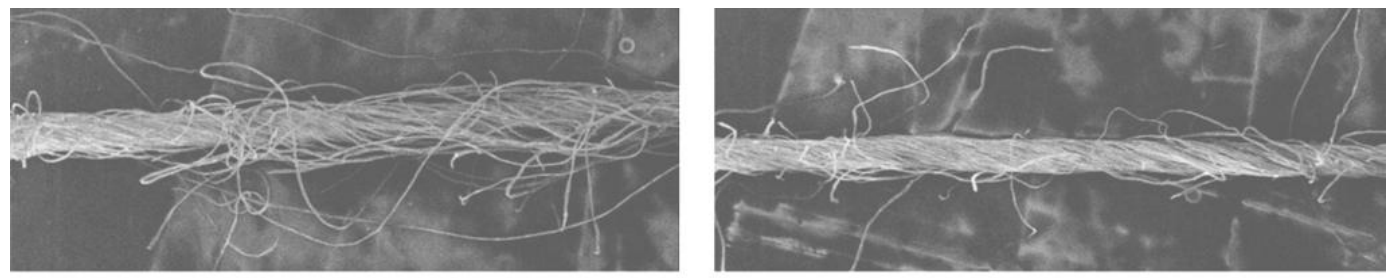

Fig 15 - Un-treated 30 Ne Regular Yarn

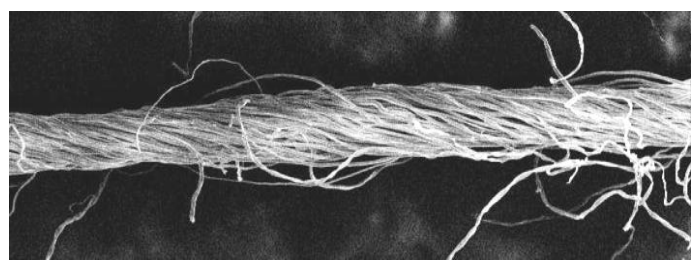

Fig 16 - Un-treated 30 Ne Compact Yarn

After normalisation, it is noticed that in respect of compact yarns, all the treatments have led to an increase in the energy. In the case of regular yarns, bleaching with hydrogen peroxide has improved compression energy. Surprisingly, slack mercerisation treatment has no effect on the compression of yarns.

$\mathrm{T}_{\mathrm{m}}$ value shows some interesting features in that for compact control it is lower as compared to the regular control yarns. $\mathrm{RC}$ values do not show any consistent trend. With regard to the compact dyed yarn the value had risen sharply to $53.8 \%$. Hydrogen peroxide treatment had reduced the value to $25.64 \%$ when compared to the control $(35.56 \%)$.

Fig 17 - Slack Mercerised 30 Ne Regular Yarn Fig 18 - Slack Mercerised 30 Ne Compact Yarn

The scanning electron micrograph pictures (Figures $15-18$ ) presented show the features that are present in regular and compact yarns ${ }^{[20]}$ It is quite clear that the compact yarn has less number of hairs on its surface than the regular yarn. The scanning electron micrographs of slack mercerised yarn show very clearly the increase in bulk due to slack mercerization treatment.

\section{CONCLUSIONS}

The following conclusions can be drawn from the above studies:

- $\quad$ The compact untreated and treated yarns are stronger than those of the regular yarns. Finishing treatments influenced the strength of the yarns. Treatments like scouring and mercerisation improved the tenacity of the yarns. Bleaching treatments reduced the yarn

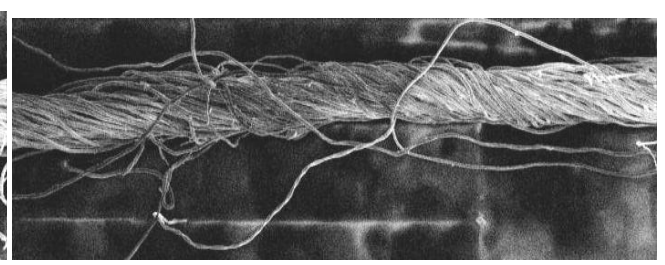

tenacity, the peroxide bleach affected the compact yarn more than the regular yarn. Dyeing improved the tenacity of compact yarn vis - a - vis regular yarn.

- The compact yarn samples exhibited better elongation values than those of the regular yarns in some cases and vice versa. The slack mercerisation treatment increased the elongation percentage of both the yarns. Treatments like scouring, bleaching and dyeing reduced the yarn elongation percentage.

- $\quad$ The compact yarn had better work of rupture values when compared to the regular yarn. The scouring, mercerisation and dyeing treatments improved the work of rupture value of compact yarn. Treatments such as bleaching reduced the values, while the reduction due to peroxide bleach was significant. 
- $\quad$ The untreated and the treated compact yarns had higher initial moduli than their counterparts. While the value decreased significantly after mercerisation, the value increased following bleaching and dyeing.

- $\quad$ The abrasion resistance of compact yarn increased following most of the treatments except for peroxide bleach.

- $\quad$ Yarn friction values of both the compact and regular yarns increased following the treatments. The increase was significantly higher in the case of all the compact yarns than their regular yarn counterparts.

- $\quad$ All the chemical treatments caused shrinkage in both the yarns. The shrinkage caused by bleaching, mercerisation and dyeing was more pronounced than that caused by scouring. The regular yarns exhibited more shrinkage than compact yarns.

- All the treatments have resulted in reduction of compression percentage in respect of compact yarns. The regular yarn compression percentage was found to decrease following scouring and dyeing, while the other treatments improved its compression percentage.

- $\quad$ The compression resilience is highly correlated with the yarn thickness $\left(\mathrm{T}_{\mathrm{m}}\right)$.

\section{REFERENCES}

[1] Salhotra K.R. Spinning of Man-Made and Blends on Cotton Systems, The Textile Association of India Publication, 2004.

[2] Sevda Altas and Hüseyin Kadoğlu, Comparison of Conventional Ring, Mechanical Compact and Pneumatic Compact Yarn Spinning Systems, Journal of Engineered Fibers and Fabrics, Volume 7, Issue 1, Pg 87 http://www.jeffjournal.org, 2012.

[3] Ömeroğlu S. and S. Ülkü, An Investigation about Tensile Strength, Pilling and Abrasion Properties of Woven Fabrics Made from Conventional and Compact Ring-Spun Yarns, Fibers \& Textiles in Eastern Europe, 15(1), p. 39-42, 2007.

[4] Kampen W., The Advantages of Condensed Spinning, Melliand English, No.4, p.58-59, 2000.

[5] Tadeusz Jackowski, Danuta Cyniak and Jerzy Czekalski, Compact Cotton Yarn, Fibres \& Textiles in Eastern Europe, October / December, Vol. 12, No. 4 (48) Pg 23, 2004.

[6] Subramanian S.N., Venkatachalam A. and Subramanian V., 'A Comparative Study of the Characteristics of Regular, Compact and Double Yarns', $17^{\text {th }}$ National Convention \& All India Seminar in Innovation in textile Process and Products, Coimbatore, p. 28-31, 2005.

[7] Meyer U., Compact Yarns: innovation As a Sector Driving Force, Melliand International, 6, March, p. 2, 2000.

[8] Celik P. and Kadoglu H., A Research on the Compact Spinning for Long Staple Yarns, Text. Res. J., 12/4, p. 27-31, 2004.
[9] Gahlert E., Asal R. and Hellwig A., Using Com4 Yarns in Piece-Dyed Shirting Fabrics, Melliand Textileberichte, 84/11-12, p. 171-172, 2003.

[10] Barella A., Vigo J.P. and Esperon H.O., An Application of Mini computers to the Optimization of the Open End Spinning Process Part-I: Consideration of Case of Two Variables, J. Text. Inst., Vol. 67, p. 253-260, 1976.

[11] Tripp W.V., Ziffle H.M. and Conard C.M., A Study on the Effect of Mercerisation on Yarn Properties, Text. Res. J., No. 404, p. 28, 1958.

[12] Cheek L. and Roussel L., Merceriastion of Ramie: Comparisons with Flax and Cotton, Text. Res. J., Vol. 59, No. 8, p. 478-483, 1989.

[13] Rebenfeld L., Response of cotton Treatments Part-I: Fiber Mercerisation and Urea Treatments 1, Text. Res. J., Vol. 31, No.2, p. 123-130, 1961.

[14] Nayar C. R., An Evaluation of the Effect of Machine Settings and Construction Parameters on Open End Yarn Properties, Doctoral Thesis, Anna University, Chennai, 1991.

[15] Karthikeyan A., A study on Mercerisation on Open End and Ring Spun Cotton Yarns by Conventional and Foam Technology Methods, M.Sc Research Thesis, Anna University, Chennai, 1984.

[16] Imayathanizhan and Gajendra, An Investigation of Mixed Fibre Quality by Condensing the Mass of Kapos Seed and Locules of Different Cotton Verities, Asian Textile J., Vol. 31, p. 62-69, 2007.

[17] Binternagel T., New Potential in Downstream Processing of Com4 Yarns, Melliand International, p. 161-162, 2000.

[18] Ragunathan K., An Investigation on the Low Stress Mechanical Properties of Rotar Yarns and Ring Yarns, Doctoral Thesis, Anna University, Chennai, 1998.

[19] Kawabata S., The Standardisation and Analysis of Hand Evaluation, The Textile Machinery Society of Japan, Osaka, Japan, (1982).

[20] Afrose Fathima Farid, A Study of Low Stress Mechanical Properties of Fabrics Produced by Different Spinning Technologies, Ph.D. Thesis, University of Madras, Chennai, 2007. 